\title{
Two Case Studies Illustrating How Relatively Selective Hippocampal Lesions in Humans Can Have Quite Different Effects on Memory
}

\author{
J.S. Holdstock, ${ }^{1 *}$ D.M. Parslow, ${ }^{2}$ R.G. Morris, ${ }^{2}$ S. Fleminger, ${ }^{3}$ S. Abrahams ${ }^{4}$ C. Denby, ${ }^{5}$ \\ D. Montaldi, ${ }^{5}$ and A.R. Mayes ${ }^{5}$
}

\begin{abstract}
Two patients, with magnetic resonance imaging (MRI)confirmed relatively selective hippocampal damage, showed distinct patterns of performance on tests of recall, item recognition, and associative recognition. Patient $\mathrm{AC}$ showed a mean bilateral volume reduction of the hippocampus of $28 \%$, but displayed no memory deficit. Both recall and recognition memory were unimpaired. In contrast, patient PR, who showed a mean bilateral hippocampal volume reduction of $59 \%$, was more consistently impaired on recall than recognition tests, although his recognition scores were highly variable. Patients $\mathrm{AC}$ and PR illustrate how variable the memory deficit following seemingly selective hippocampal damage can be in humans. They highlight the need for more sophisticated imaging in future studies if the human hippocampus' role in memory is to be fully identified. $\odot 2008$ Wiley-Liss, Inc.
\end{abstract}

KEY WORDS: recognition; recall; familiarity; recollection; amnesia

\section{INTRODUCTION}

The medial temporal lobe is a region of the brain that has been known for many years to play a critical role in memory (e.g., Scoville and Milner, 1957). The prevailing view has been that the hippocampus and the adjacent perirhinal, entorhinal, and parahippocampal cortices, which together comprise the medial temporal lobe, work as a single memory system to support both recall and recognition memory (Squire and Zola-Morgan, 1991). However, recent electrophysiological and lesion studies from the animal literature led Aggleton and Brown (1999) to challenge this view. They argued that two systems involving medial temporal lobe and diencephalic structures can be distinguished and that each of these supports a different type of memory. Drawing on dual process models of recognition memory (e.g., Mandler, 1980; Jacoby, 1991), Aggleton and Brown have argued that a system comprising primarily hippocampus, fornix, and anterior thalamus, with inputs from

\footnotetext{
${ }^{1}$ School of Psychology, University of Liverpool, Liverpool, United Kingdom; ${ }^{2}$ Department of Psychology, King's College, Institute of Psychiatry, University of London, De Crespigny Park, London, United Kingdom; ${ }^{3}$ Lishman Brain Injury Unit, Maudsley Hospital, London, United Kingdom; ${ }^{4}$ School of Philosophy, Psychology and Language Sciences, The University of Edinburgh, Teviot Place, Edinburgh, United Kingdom; ${ }^{5}$ School of Psychological Sciences, University of Manchester, Oxford Road, Manchester, United Kingdom

*Correspondence to: Juliet S. Holdstock, School of Psychology, University of Liverpool, Eleanor Rathbone Building, P.O. Box 147, Liverpool, L69 3BS, UK. E-mail: juliet@julietholdstock.com

Accepted for publication 30 January 2008

DOI 10.1002/hipo.20427

Published online 8 April 2008 in Wiley InterScience (www.interscience. wiley.com).
}

entorhinal cortex (EC) and connections with frontal cortices, supports recall and recollection (the cued recall of specific information about a study event that is used in making a recognition decision). In contrast, a second system, which comprises primarily perirhinal cortex, dorsomedial thalamus, and frontal cortices, supports recognition memory when this can be based on familiarity (a feeling that reencountered information has been experienced previously that occurs in the absence of any recall).

These two theoretical views make very different predictions about the effect of selective hippocampal damage on memory. The view of Squire and ZolaMorgan predicts that both recall and recognition will be equally impaired whereas the view of Aggleton and Brown predicts that recognition will be more spared than recall, and that the degree of relative sparing will depend on the extent to which familiarity is sufficient to support normal levels of recognition.

The small number of investigations of the effect of hippocampal lesions in humans have produced conflicting findings. Consistent with the view of Aggleton and Brown, some patients have been reported to show unimpaired performance on item recognition tests despite impaired recall and impaired recognition of at least some kinds of arbitrary associations (Vargha-Khadem et al., 1997; Henke et al., 1999; Mayes et al., 2002; Bastin et al., 2004; Mayes et al., 2004; Turriziani et al., 2004; Aggleton et al., 2005; Barbeau et al., 2005; Holdstock et al., 2005). Furthermore, familiarity was shown to be intact in a number of these patients (Holdstock et al., 2002a; Bastin et al., 2004; Aggleton et al., 2005; Barbeau et al., 2005). Familiarity was also shown to be preserved in a group of hypoxic patients with presumed hippocampal atrophy, who showed a greater impairment of recall than recognition memory (Yonelinas et al., 2002). In contrast, and consistent with the view of Squire and Zola-Morgan, other patients have been reported to have approximately equally impaired item recognition, recall, and associative recognition (e.g., Kartsounis et al., 1995; Reed and Squire, 1997; Cipolotti et al., 2001; Stark et al., 2002; Stark and Squire, 2003; Cipolotti et al., 2006; Gold et al., 2006). These patients have also been shown to be impaired at both familiarity and recollection (Manns et al., 2003; Cipolotti et al., 2006; Wais et al., 2006). However, it should be noted 
that for one of these patients, VC, face recognition has been reported to be spared. His recollection and familiarity for faces was preserved despite recognition deficits for other materials (Cipolotti et al., 2006). Sparing of face recognition after hippocampal damage has also been recently reported for other patients (Lee et al., 2005; Graham et al., 2006; Bird et al., 2007, in press).

In the present article, we describe the item recognition, recall, and associative recognition performance of two further patients for whom detailed volumetric analysis of structural MRI suggests selective hippocampal atrophy. These two patients suffered hippocampal damage as relatively young adults (aged 39 and $24 \mathrm{yr}$ ), which makes them of particular interest because of the lack of complications associated with age related brain changes that can be an issue with older patients. The patterns of performance shown by these two patients are different to those reported in previous cases and illustrate further the heterogeneity of memory impairments that can result from seemingly selective hippocampal damage in humans.

\section{METHODS}

\section{Participants}

Approval for this study was provided by the Institute of Psychiatry and South London and Maudsley NHS Trust Ethical Committee. All subjects gave informed consent before taking part in the study.

Two patients, AC and PR, participated in the study. The inclusion of these patients was based purely on the results of structural MRI analyses. Both patients showed significant, and apparently selective, bilateral hippocampal volume reductions relative to matched healthy control subjects. The presence of amnesia at the time of testing was not an inclusion criterion for this study.

\section{Case AC}

$\mathrm{AC}$ was an electrical engineer who suffered an anoxic brain injury in 1997. His brain injury followed a complicated splenectomy and pancreatectomy during which he also had a grand mal epileptic seizure with status. He remained fully unconscious for 2 weeks and had a period of anterograde amnesia for a further 2 weeks. He was then admitted to a brain injury unit for a neurorehabilitation program and he had difficulties with memory and sensory motor functioning. After about 6 months the sensory motor dysfunction had resolved and he had residual slowed information processing and mild memory impairment. At the time of his inclusion in the study (about 5 yr later; aged $44 \mathrm{yr}$ ) he did not show information processing reduction and he did not complain of memory difficulties.

At the time of testing, AC's Full-Scale IQ was estimated to be 131, using the Wechsler Abbreviated Scale of Intelligence
(WASI) (1999). On the Doors and People Test (Baddeley et al., 1994), which measures recall and recognition of verbal and visual information, AC's performance was unimpaired. His performance was comparable for recall and recognition, although there was a trend for slightly lower performance for recall (scaled scores of 12 (75th percentile) for both People (verbal recall) and Shapes (visual recall) compared with 16 (95th percentile) and 14 (90th percentile) for Names (verbal recognition) and Doors (visual recognition), respectively).

\section{Case PR}

Patient PR suffered anoxic brain damage at the age of $24 \mathrm{yr}$ and was $32 \mathrm{yr}$ old when participating in the study reported here. He has been reported previously, in relation to his spatial memory abilities, by Parslow et al. (2005). PR suffered carbon monoxide poisoning as a result of a suicide attempt following three periods of major depression. As a result of this he sustained anoxic brain damage, which resulted in a specific memory deficit. He complains of forgetfulness and difficulty taking in new information but reports having intact remote memories preceding the incident. PR's depression stabilized after his anoxic brain damage and assessment by a consultant neuropsychiatrist found that he was not depressed at the time of the study.

At the time of testing, his Full-Scale IQ measured using the WASI was 129. On the Doors and People Test, PR was clearly impaired at recall, obtaining scaled scores of 5 ( 5 th percentile) and $1 \quad(<1$ st percentile) for the People (verbal recall) and Shapes (visual recall) subtests, respectively, but not at recognition, obtaining scaled scores of 15 (90th percentile) and 12 (75th percentile) for the Names (verbal recognition) and Doors (visual recognition) subtests, respectively. PR's recall of visual and verbal information has also been reported to be impaired on the Adult Memory Information Processing Battery (AMIPB) (Parslow et al., 2005). In contrast, PR's executive functions were reported to be intact (Parslow et al., 2005). PR obtained $112 / 112$ on the Stroop test and performed at between the 75 th and 90th percentile on both parts A and B of the Trail Making Test (Parslow et al., 2005).

\section{Control Subjects}

The performance of the patients was compared with that of a group of 10 age- and IQ-matched male controls who were employees of the Institute of Psychiatry, London. The mean age of the control group was $37 \mathrm{yr}(\mathrm{SD}=5.52)$ with a mean WASI IQ of 124.5 (SD = 7.706). Both patients had WASI full scale IQs that were slightly higher than the control mean but which did not differ significantly from it (Crawford and Howell (1998) modified $t=0.804, P=0.442$ for AC; modified $t=0.557, P=0.591$ for PR). The age of the patients did not differ significantly from the control mean (Crawford and Howell (1998) modified $t=1.190, P=0.264$ for AC; modified $t=-0.880, P=0.402$ for PR). 


\section{MRI Investigation}

\section{Scan acquisition}

Structural MRI scans were obtained for AC, PR and a further comparison set of 10 healthy age matched controls, using a 1.5T GE NV/I Signa System (General Electric, Milwaukee, WI) (Maudsley Hospital, London, UK).

For volumetric measurements T1-weighted 3-D inversion recovery images were obtained in the coronal plane, orientated perpendicular to the line of the hippocampus with $1.5 \mathrm{~mm}$ contiguous sections $(\mathrm{TR}=1.8 \mathrm{~ms}$, $\mathrm{TI}=450 \mathrm{~ms}, \mathrm{TE}=5.1$ ms, flip angle $=20^{\circ}, 256 \times 256 \times 128$ pixel matrix, $24 \mathrm{~cm}$ field of view, slice thickness $=1.5 \mathrm{~mm}$, one data average, acquisition time $=8.21$.

\section{Volume Estimation}

Volumetric analyses were performed on AC, PR and the 10 controls using the disprim image display (David Plummer, Medical Physics Department University College London: http://www.medphys.ucl.ac.uk). The regions of interest were demarcated using a mouse-driven manual draw facility and areas in $\mathrm{mm}^{2}$ automatically calculated. Measurements of the hippocampus and cerebral hemispheres were obtained using methods adapted from Abrahams et al. (1999). Measurements of the perirhinal and entorhinal cortices were based on the methods described by Insausti et al. (1998). Measures were duplicated for PR and one control participant to check the reliability of measurement. Chronbach's alpha was computed for each brain region and the resulting coefficients varied between 0.89 and 0.99 .

The following anatomical landmarks were used to define the structures of interest.

Hippocampus. This included the dentate gyrus (DG), the hippocampus proper, the subiculum but not the fimbria. The ventral boundary was the white/gray matter of the hippocampal formation and the parahippocampal gyrus. Measurements began on slices at the anterior end of the hippocampus where there was clear separation between the hippocampus and the amygdala and proceeded posteriorly for 15 slices (see Abrahams et al., 1999 for more details of the technique used).

Perirbinal cortex. The perirhinal and entorhinal cortices were measured using the technique developed by Insausti et al. (1998). The perirhinal cortex measurements began at the anterior end in a coronal slice just anterior to the limen insulae. At the point that the EC appears it was measured within the banks of the collateral sulcus, being approximately half way along the medial bank and taking up the lateral bank completely. The posterior end was marked by the appearance of the gyrus limbicus.
Entorbinal cortex. Measurement within this structure began in slices just posterior to the limen insulae and bordering with the perirhinal and amygdaloid cortices. The end of the EC was about $1 \mathrm{~cm}$ behind the most posterior portion of the uncus, the gyrus intralimbicus. Laterally, the EC extends into the medial bank of the collateral sulcus, the extent depending on depth of the sulcus (see Insausti et al., 1998, for more detail).

Parabippocampal cortex. This structure was defined as the part of the parahippocampal gyrus posterior to where the posterior perirhinal cortices disappeared. Accordingly, measurements began in slices posterior to the perirhinal cortices and were conducted using the method described by Abrahams et al. (1999) to measure the entire parahippocampal gyrus. The measurements followed the gray/white matter of the border between the hippocampal formation and the parahippocampal gyrus, and included the mesial bank of the collateral sulcus.

Cerebral hemispheres. This area included the whole hemisphere excluding the pons, cerebellum and brain stem, and excluding the hippocampal formation and parahippocampal gyrus. The same 15 slices as used in the hippocampal formation measurements were taken.

\section{Memory Tests}

The participants were tested on a battery of tests comprising recall, item recognition, and associative recognition for verbal and nonverbal material.

The recall tests were as follows: (1) Recalling a short story consisting of a single paragraph of information after filled delays of $20 \mathrm{~s}$ and $10 \mathrm{~min}$ (using the materials of Isaac and Mayes, 1999). (2) Recalling the identity of 12 line-drawn pictures of objects after filled delays of $40 \mathrm{~s}$ and $30 \mathrm{~min}$ (separate sets of objects were used for each delay). This task is described in detail by Holdstock et al. (2002a). (3) Recalling the temporal order of words and patterns. On each trial participants had to recall, after a filled $15 \mathrm{~s}$ delay, the order in which eight stimuli (words in one task and patterns in the other) were presented. These tasks are described in detail by Mayes et al. (2001, experiment 2). (4) Recalling 10 definitions cued, in each case, by the word with which it was paired at study. The words and definitions used in this test were unfamiliar to the subjects and cued recall was tested after each of 10 learning trials. This task is described in detail by Holdstock et al. (2002b, experiment 1).

Item recognition was assessed using the following tests: (1) Four choice forced-choice recognition of facts from a short story, consisting of a single paragraph, after filled delays of $20 \mathrm{~s}$ and $10 \mathrm{~min}$ (see Isaac and Mayes, 1999). (2) Four choice forced-choice recognition and yes/no recognition of line-drawn pictures of objects after delays of $40 \mathrm{~s}$ and $30 \mathrm{~min}$. These tests required a very fine discrimination between target and foils. Separate sets of stimuli were used for each task and each delay. These tasks are described in detail by Holdstock et al. (2002a). 
(3) Five choice forced-choice and yes/no recognition of words and patterns (similar to those seen on wallpaper) after a delay of 15 s (see Mayes et al., 2001, experiment 2). (4) Four choice forced-choice recognition of previously studied definitions (which had been novel to the participant prior to the study) after delays of $0 \mathrm{~s}$ and $30 \mathrm{~min}$. This test is described in detail by Holdstock et al. (2002b, experiment 1).

The associative recognition tasks involved recognition of the temporal order in which words and patterns were presented, and recognition of pairings of words and definitions that had been novel to the participant prior to the study. The temporal order tasks are described in detail by Mayes et al. (2001, experiment 2). On each trial participants studied five new stimuli (patterns in one task, words in the other task) and were instructed to remember both the stimuli and the order in which they were presented. At test, which followed a $15 \mathrm{~s}$ filled delay, participants were shown the studied stimuli in five different orders and they had to decide which of these corresponded to the order in which they had been presented at study. The word-definition pair recognition task required participants to study 10 word-definition pairs over 10 learning trials and then, immediately following the tenth learning trial and after a 30 min delay, required participants to select, for each of the stud- ied words, which of four studied definitions had been paired with it. This task is described in detail by Holdstock et al. (2002b, experiment 1). These associative recognition tests all used recombination foils (i.e., the foils were new, unstudied, combinations of studied components) to ensure that subjects had to use their memory for the associations between the components and could not rely on their memory for the individual components themselves.

\section{RESULTS}

\section{Neuroimaging Results}

The estimated volumes of the hippocampus, perirhinal cortex, EC, parahippocampal cortex, and cerebral hemispheres are shown in Table 1 for AC, PR, and the 10 control subjects. The structure volumes for the two patients and controls were normalized for intracranial volume using the formula of Jack et al. (1989). This adjusted the observed structure volumes by an amount that was proportional to the difference between an individual's observed total intracranial volume and the mean in-

TABLE 1.

Actual Volumes $\left(\mathrm{mm}^{3}\right)$, and Volumes Normalized for Intracranial Volume, of Hippocampus, Perirhinal Cortex, Entorhinal Cortex, Parahippocampal Cortex, and Cerebral hemispheres for AC, PR, and 10 Matched Controls

\begin{tabular}{|c|c|c|c|c|c|}
\hline & $\begin{array}{c}\text { Control mean } \\
\text { (SD actual volume) } \\
\text { (SD normalized volume) }\end{array}$ & $\begin{array}{c}\text { AC actual } \\
\text { volume }\end{array}$ & $\begin{array}{c}\mathrm{AC} \\
\text { normalized } \\
\text { volume }\end{array}$ & $\begin{array}{c}\text { PR actual } \\
\text { volume }\end{array}$ & $\begin{array}{c}\text { PR } \\
\text { normalized } \\
\text { volume }\end{array}$ \\
\hline \multirow[t]{2}{*}{ Right hippocampus } & 2927.88 (170.68) & $2019.45^{*}$ & $2018.55^{*}$ & $1270.65^{*}$ & $1264.94^{*}$ \\
\hline & $(170.22)$ & $z=-5.32$ & $z=-5.34$ & $z=-9.71$ & $z=-9.77$ \\
\hline \multirow[t]{2}{*}{ Left hippocampus } & $2792.06(194.45)$ & $2094.75^{*}$ & $2095.65^{*}$ & $1095.60^{*}$ & $1101.31^{*}$ \\
\hline & (194.35) & $z=-3.59$ & $z=-3.58$ & $z=-8.72$ & $z=-8.70$ \\
\hline \multirow[t]{2}{*}{ Right entorhinal cortex } & 1986.98 (305.93) & 1621.20 & 1619.40 & 2006.40 & 1994.99 \\
\hline & $(305.28)$ & $z=-1.20$ & $z=-1.20$ & $z=0.06$ & $z=0.03$ \\
\hline \multirow[t]{2}{*}{ Left entorhinal cortex } & $2001.63(282.23)$ & 1821.60 & 1818.01 & 1943.25 & 1920.43 \\
\hline & $(279.73)$ & $z=-0.64$ & $z=-0.66$ & $z=-0.21$ & $z=-0.29$ \\
\hline \multirow[t]{2}{*}{ Right perirhinal cortex } & $4928.96(1155.50)$ & 3870.60 & 3939.79 & 3026.10 & 3465.40 \\
\hline & $(863.49)$ & $z=-0.92$ & $z=-1.15$ & $z=-1.65$ & $z=-1.69$ \\
\hline \multirow[t]{2}{*}{ Left perirhinal cortex } & 4109.31 (1045.70) & 4568.10 & 4596.86 & 3444.75 & 3627.32 \\
\hline & $(996.11)$ & $z=0.44$ & $z=0.49$ & $z=-0.64$ & $z=-0.48$ \\
\hline \multirow[t]{2}{*}{ Right parahippocampal cortex } & $1337.46(469.26)$ & 1698.00 & 1706.09 & 1190.25 & 1241.60 \\
\hline & $(460.80)$ & $z=0.77$ & $z=0.80$ & $z=-0.31$ & $z=-0.21$ \\
\hline \multirow[t]{2}{*}{ Left parahippocampal cortex } & $1384.02(366.55)$ & 1909.05 & 1926.12 & 1024.05 & 1132.45 \\
\hline & $(313.73)$ & $z=1.43$ & $z=1.73$ & $z=-0.98$ & $z=-0.80$ \\
\hline \multirow[t]{2}{*}{ Right cerebral hemisphere } & $118848.8(5223.34)$ & 117364.80 & 117778.16 & 113004.60 & 115628.99 \\
\hline & $(2506.46)$ & $z=-0.28$ & $z=-0.43$ & $z=-1.12$ & $z=-1.28$ \\
\hline \multirow[t]{2}{*}{ Left cerebral hemisphere } & 119190.9 (5407.20) & 113396.10 & 113825.63 & 111220.80 & 113947.89 \\
\hline & $(2565.12)$ & $z=-1.07$ & $z=-2.09$ & $z=-1.47$ & $z=-2.04$ \\
\hline \multirow[t]{2}{*}{ Intracranial volume } & 257889.1 (9951.88) & 256990.50 & $\mathrm{~N} / \mathrm{A}$ & 252183.90 & $\mathrm{~N} / \mathrm{A}$ \\
\hline & & $z=-0.09$ & & $z=-0.57$ & \\
\hline
\end{tabular}

The control mean is the same for both actual and normalized volumes.

${ }^{*} P<0.05$, two-tailed, when compared with the control group using a modified $t$-test (Crawford and Howell, 1998). 


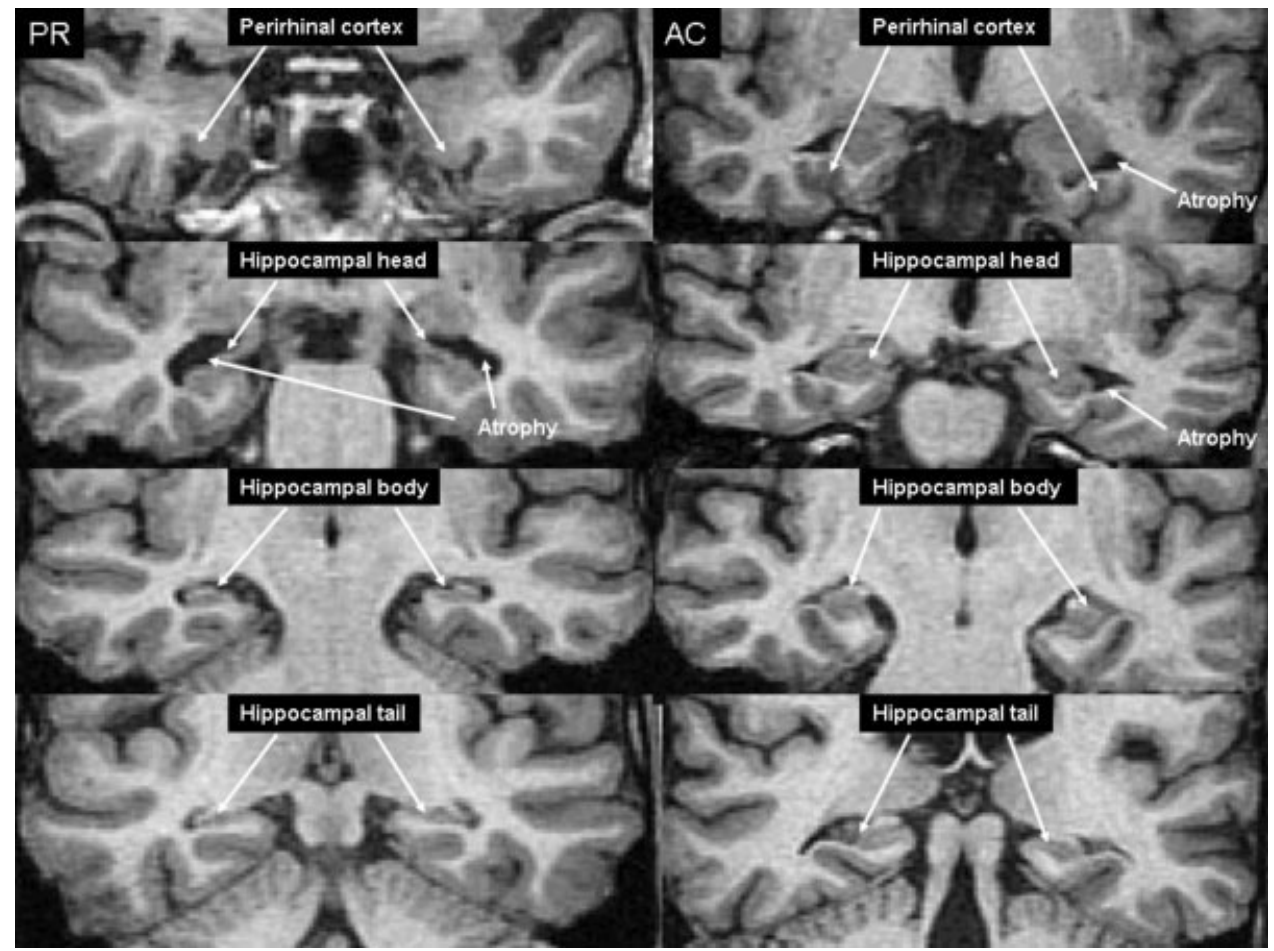

FIGURE 1. T1 weighted coronal images through the temporal lobe showing hippocampal atrophy in patients PR and AC. Hippocampal atrophy is present in the head of the hippocampus in both subjects. Patient PR displays severe bilateral atrophy across the entire hippocampal formation, in comparison with AC where atro- phy is mainly apparent in the amygdala and hippocampal head, although the tail of the right hippocampus also looks unusual in this patient. Abnormality could not be detected in the perirhinal cortex of either patient by an experienced neuroradiologist (Joe Jarosz, personal communication). tracranial volume for the control group. We present both actual and normalized structure volumes in Table 1 . The data for each patient were analyzed separately. To compare the patient's normalized volumes of each measured brain structure with the volumes of these structures in the control group, we used a modified $t$-test. This test allowed an individual's score to be compared against a small control sample (Crawford and Howell, 1998). The test was implemented using the program SINGLIMS.EXE (Crawford and Garthwaite, 2002).

For both patients, we found significant volume reductions in the left and right hippocampus only. As can be seen in Table 1, there was a greater reduction in hippocampal volume for PR than AC. For PR, normalized hippocampal volume was reduced by $56.8 \%$ in the right hippocampus and $60.6 \%$ in the left hippocampus. The substantial nature of PR's hippocampal atrophy is consistent with anoxic damage rather than the effects of depression, which has been associated with subtle reductions in hippocampal volume (of between 8 and 19\%) and function (Sheline et al., 2002). However, it is possible that changes in hippocampal structure or function, associated with depression, before anoxia may have contributed to his resulting lesion. For $\mathrm{AC}$, right normalized hippocampal volume was reduced by $31.1 \%$ and left normalized hippocampal volume by $24.9 \%$. Figure 1 shows T1-weighted MR images of the hippocampus for PR and AC.
Although there were no significant reductions in the volumes of the other structures we measured, inspection of Table 1 showed that PR's perirhinal cortex was smaller than the control mean bilaterally with the volume difference being greater on the right. His right perirhinal cortex, corrected for intracranial volume, was 1.69 SDs below the control mean (30\% smaller than the control mean) whereas his left perirhinal cortex was 0.48 SDs below the control mean (12\% smaller than the control mean). For both patients, the volume of the left cerebral hemisphere was 2 SDs below the control mean.

\section{Memory Test Results}

The memory test data from the two patients were analyzed as two single case studies. In each case, the patient's performance on each test was compared with the performance of the control group using modified $t$-tests (Crawford and Howell, 1998; using SINGLIMS.EXE Crawford and Garthwaite, 2002). Results for the individual tests are shown in Table 2 for both patients. For the two temporal order recall tasks the correlation between recalled order and the actual studied order was calculated and used as the measure of memory. For all other recall tasks and for the forced-choice recogni- 
TABLE 2.

Scores of Patients AC and PR and a Group of Matched Control Subjects on Tests of Recall and Recognition Memory

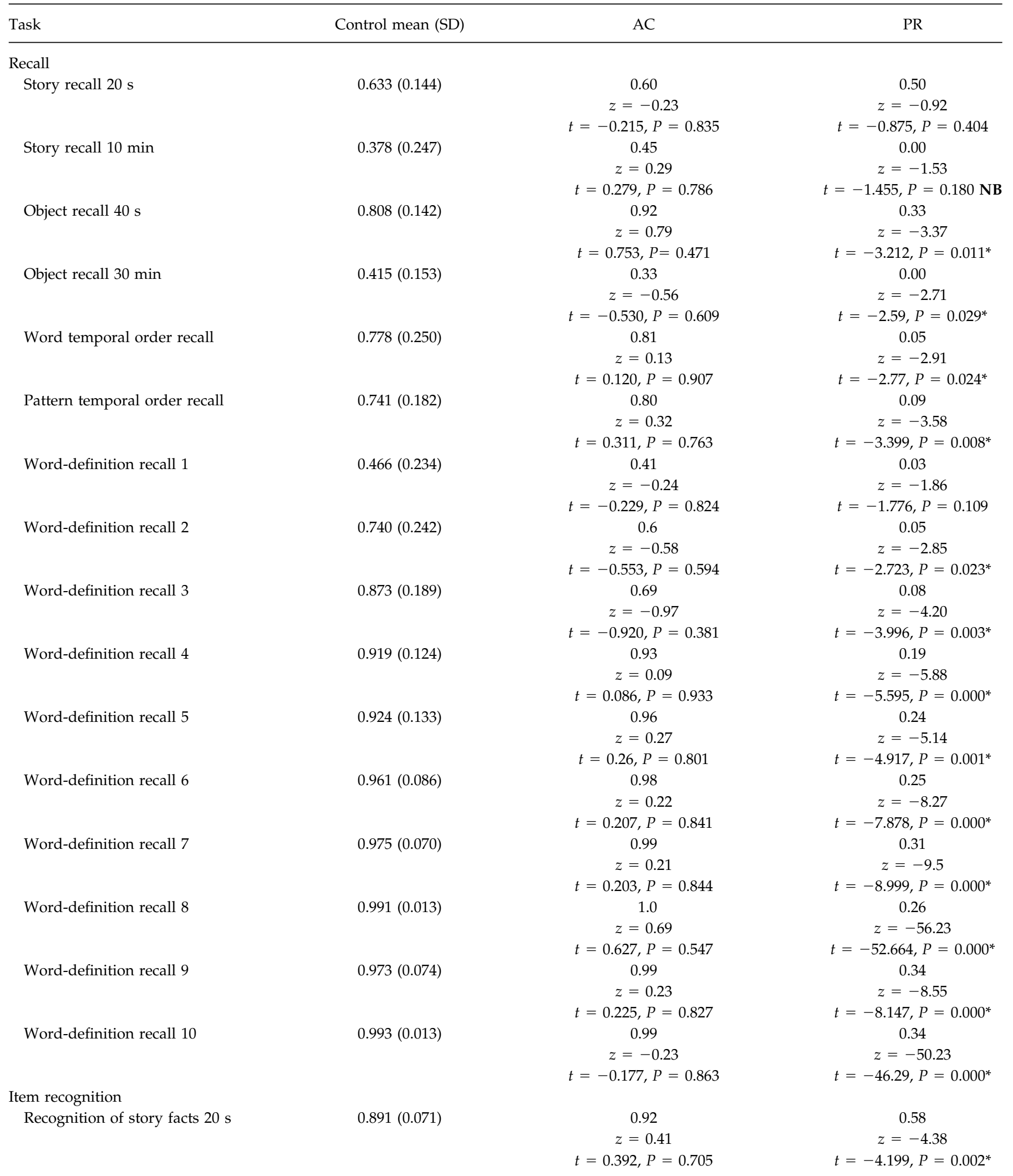


TABLE 2.

(Continued)

\begin{tabular}{|c|c|c|c|}
\hline Task & Control mean (SD) & $\mathrm{AC}$ & PR \\
\hline Recognition of story facts $10 \mathrm{~min}$ & $0.718(0.041)$ & $\begin{array}{c}0.58 \\
z=-3.37 \\
t=-3.185, P=0.011^{*}\end{array}$ & $\begin{array}{c}0.25 \\
z=-11.41 \\
t=-10.802, P=0.000^{*}\end{array}$ \\
\hline Forced-choice object recognition $40 \mathrm{~s}$ & $0.676(0.149)$ & $\begin{array}{c}0.5 \\
z=-1.18 \\
t=-1.126, P=0.289\end{array}$ & $\begin{array}{c}0.5 \\
z=-1.18 \\
t=-1.126, P=0.289\end{array}$ \\
\hline Forced-choice object recognition $30 \mathrm{~min}$ & $0.751(0.137)$ & $\begin{array}{c}0.67 \\
z=-0.59 \\
t=-0.562, P=0.588\end{array}$ & $\begin{array}{c}0.25 \\
z=-3.66 \\
t=-3.475, P=0.007^{*}\end{array}$ \\
\hline Yes-no object recognition $40 \mathrm{~s}$ & $0.809(0.065)$ & $\begin{array}{c}0.59 \\
z=-3.37 \\
t=-3.212, P=0.011^{*}\end{array}$ & $\begin{array}{c}0.57 \\
z=-3.68 \\
t=-3.506, P=0.007^{*}\end{array}$ \\
\hline Yes - no object recognition $30 \mathrm{~min}$ & $0.822(0.104)$ & $\begin{array}{c}0.61 \\
z=-2.04 \\
t=-1.944, P=0.084\end{array}$ & $\begin{array}{c}0.75 \\
z=-0.69 \\
t=-0.660, P=0.526\end{array}$ \\
\hline Forced-choice word recognition & $0.880(0.092)$ & $\begin{array}{c}0.90 \\
z=0.22 \\
t=0.208, P=0.840\end{array}$ & $\begin{array}{c}0.50 \\
z=-4.13 \\
t=-3.943, P=0.003^{*}\end{array}$ \\
\hline Yes - no word recognition & $0.947(0.036)$ & $\begin{array}{c}0.94 \\
z=-0.19 \\
t=-0.183, P=0.860\end{array}$ & $\begin{array}{c}0.89 \\
z=-1.58 \\
t=-1.493, P=0.179\end{array}$ \\
\hline Forced-choice pattern recognition & $0.870(0.116)$ & $\begin{array}{c}0.90 \\
z=0.26 \\
t=0.247, P=0.811\end{array}$ & $\begin{array}{c}0.70 \\
z=-1.47 \\
t=-1.398, P=0.196\end{array}$ \\
\hline Yes-no pattern recognition & $0.840(0.079)$ & $\begin{array}{c}0.91 \\
z=0.89 \\
t=0.845, P=0.420\end{array}$ & $\begin{array}{c}0.22 \\
z=-7.85 \\
t=-7.483, P=0.000^{*}\end{array}$ \\
\hline Definition recognition $0 \mathrm{~s}$ & $0.890(0.110)$ & $\begin{array}{c}0.70 \\
z=-1.73 \\
t=-1.646, P=0.134\end{array}$ & $\begin{array}{c}0.80 \\
z=-0.82 \\
t=-0.780, P=0.456\end{array}$ \\
\hline Definition recognition $30 \mathrm{~min}$ & $0.650(0.212)$ & $\begin{array}{c}0.70 \\
z=0.24 \\
t=0.225, P=0.827\end{array}$ & $\begin{array}{c}0.40 \\
z=-1.18 \\
t=-1.124, P=0.290\end{array}$ \\
\hline \multicolumn{4}{|l|}{ Associative recognition } \\
\hline Word temporal order recognition & $0.817(0.154)$ & $\begin{array}{c}0.95 \\
z=0.86 \\
t=0.821, P=0.436\end{array}$ & $\begin{array}{c}0.90 \\
z=0.54 \\
t=0.513, P=0.622\end{array}$ \\
\hline Pattern temporal order recognition & $0.760(0.126)$ & $\begin{array}{c}1.0 \\
z=1.90 \\
t=1.809, P=0.104\end{array}$ & $\begin{array}{c}0.35 \\
\mathrm{z}=-3.25 \\
t=-3.091, P=0.013^{*}\end{array}$ \\
\hline Word-definition recognition $0 \mathrm{~s}$ & $0.830(0.211)$ & $\begin{array}{c}1.0 \\
z=0.81 \\
t=0.768, P=0.462\end{array}$ & $\begin{array}{c}0.80 \\
z=-0.14 \\
t=-0.136, P=0.895\end{array}$ \\
\hline Word-definition recognition $30 \mathrm{~min}$ & $0.710(0.233)$ & $\begin{array}{c}0.80 \\
z=0.39 \\
t=0.368, P=0.721\end{array}$ & $\begin{array}{c}0.80 \\
\mathrm{z}=0.39 \\
t=0.368, P=0.721\end{array}$ \\
\hline
\end{tabular}

For the patients, their $z$-score and the results of a modified $t$-test (Crawford and Garthwaite, 1998) comparing patient and control performance is also shown for each test.

NB, patient PR recalled zero points of information about the story but was not significantly impaired because of a floor effect in the control group for this test. ${ }^{*} P<0.05$, two-tailed, when compared with the control group using a modified $t$-test (Crawford and Howell, 1998).

tion tasks the performance measure was proportion correct. For yes/no recognition tasks subjects' scores were converted to $\mathrm{A}^{\prime}$ (Pollack and Norman, 1964) to obtain a bias free mea- sure of discrimination sensitivity. We were unable to use $\mathrm{d}^{\prime}$ because a number of subjects obtained $100 \%$ hits and $0 \%$ false alarms. 


\section{Patient AC}

Patient AC was unimpaired on all recall tests and on 14 of the 16 measures of recognition. Furthermore, his performance was above the control mean on 10 of the 16 measures of recall and 9 of the 16 measures of recognition. His mean recall performance was 0.03 SDs above the control mean and his mean recognition performance was 0.41 SDs below the control mean.

\section{Patient PR}

In contrast to AC, patient PR showed a consistent impairment on the recall tests. His mean recall score based on seven tests (for the word-definition learning task only performance on the tenth learning trial was included in the mean) was 9.32 SDs below the control mean. However, this measure was highly influenced by his performance on the word-definition recall test (50.23 SDs below the control mean on the tenth learning trial). Excluding this test, his mean recall score was 2.50 SDs below the control mean.

Considering all of the recall tests, the only ones on which he was unimpaired were story recall after $20 \mathrm{~s}$ and $10 \mathrm{~min}$ delays and the first learning trial of the word-definition recall task. For two of these tasks: story recall after a $10 \mathrm{~min}$ delay and the first learning trial of the word-definition recall task, his unimpaired performance was due to floor effects in the control group. As shown in Table 2, he recalled no information about the story after the $10 \mathrm{~min}$ delay and his recall was also extremely poor for the word-definition learning task.

PR's mean recognition performance was 2.78 SDs below the control mean. However, unlike his recall performance, his performance on the recognition tests was not consistently impaired. He was impaired on seven of the 16 tests, but with no specific pattern to his performance. He was both impaired and unimpaired on forced-choice and yes-no tests, verbal and nonverbal tests, tests with short and longer delays, item and associative recognition tests. It was also not the case that he was impaired only on the more difficult tests. Using as a measure of difficulty a percent score which indicated where between chance and perfect performance the control mean fell (see Holdstock et al., 2002a), PR was found to be unimpaired on five of the eight easiest tests (difficulty score 70 and above), and on four of the eight hardest tests (difficulty score $<70$ ).

To determine whether PR's recognition performance was more variable than that of both the control subjects and patient AC, we compared the standard deviation (SD) of the patients' $z$ scores for the recognition tests with that of the control group. The same was done for the recall tests, with the word-definition recall results excluded from the analysis because of PR's extremely large $z$-scores for this task. To do this, we calculated $z$ scores for the two patients and for the individual control subjects for each recall and recognition test. Then for each individual subject we calculated the SD of their recall $z$ scores and the SD of their recognition $z$ scores. The control mean SD and the $\mathrm{SD}$ of this were then calculated for recall and recognition. This enabled us to compare the patient recall and recognition standard deviations with the control mean standard deviations.

We found that patient PR's performance was significantly more variable than that of the controls for both recall and recognition, but that this variability was much greater for recognition (2.65 SDs above the control mean for recall, 10.28 SDs above the control mean for recognition). We found that patient AC was no more variable than controls for recall $(0.81$ SDs below the control mean), but was more variable than the controls for recognition (3.21 SDs above the control mean). However, the variability in his recognition performance was much less than PR's (a difference of 7.07 SDs). Inspection of PR's scores on the individual recognition tests suggested that the particularly high variability in his performance was driven by two tests on which he performed particularly poorly: delayed story fact recognition on which he performed 11.41 SDs below the control mean and yes/no pattern recognition on which he performed 7.85 SDs below the control mean. When these recognition tests were excluded from the analysis his performance variability reduced to $3.9 \mathrm{SDs}$ greater than the control mean, which was comparable with AC.

\section{DISCUSSION}

Patients AC and PR both have damage which, according to structural MRI, appears to be restricted to the hippocampus and yet these patients showed very different patterns of memory performance. AC showed no evidence of a memory deficit, performing above the control mean on more than half of the recall and recognition tests, whereas, patient PR was consistently impaired at recall and showed variable performance on the recognition tests.

At a broad level, our findings could be considered consistent with both of the main opposing views: patient PR had a larger hippocampal lesion and poorer memory than $\mathrm{AC}$ consistent with the view that larger medial temporal lobe lesions lead to greater deficits in declarative memory (Squire and Zola-Morgan, 1991); overall PR was more impaired on recall than recognition consistent with the view that selective hippocampal lesions affect recall more than recognition (Aggleton and Brown, 1999). However, as will be apparent from the following discussion, neither theory can account for the variability in performance between patients and our data are not consistent with the more specific predictions of both theories unless additional assumptions are made, such as about the functional extent and location of the lesion or the course and extent of the recovery process.

Although different research groups have reported inconsistent patterns of memory deficits after hippocampal lesions, this is the first time that the variable effect of hippocampal lesions has been demonstrated in one study that included detailed investigation and the completion of identical tests by both patients. Furthermore, our patients showed different patterns of memory performance to the other cases that have been reported 
in the literature. This was the case even though detailed volumetric analysis of their structural MRI scans suggested comparable levels of hippocampal damage with those reported in previous cases where damage was believed to be relatively selective to the hippocampus. Taken together with previous work, our data show that the effect on memory of hippocampal lesions can vary enormously between patients, ranging from no memory deficit, as in the case of patient $\mathrm{AC}$ reported here, through a greater deficit in recall and associative recognition than item recognition memory, which is relatively spared (Vargha-Khadem et al., 1997; Henke et al., 1999; Mayes et al., 2002; Bastin et al., 2004; Turriziani et al., 2004; Aggleton et al., 2005; Barbeau et al., 2005; Holdstock et al., 2005), through consistently impaired recall but variable recognition memory, as in the case of patient PR reported here, to clearly impaired recall and recognition memory (Kartsounis et al., 1995; Reed and Squire, 1997; Cipolotti et al., 2001; Stark et al., 2002; Stark and Squire, 2003; Cipolotti et al., 2006; Gold et al., 2006). Such wide-ranging effects of hippocampal damage are not predicted by either of the two main opposing theories of hippocampal function (Squire and Zola-Morgan, 1991; Aggleton and Brown, 1999) and cannot be currently explained. In the remainder of the article, we consider PR and AC's patterns of memory performance in more detail and discuss the implications of our data for future studies.

Like a number of previously reported cases (Vargha-Khadem et al., 1997; Henke et al., 1999; Mayes et al., 2002; Bastin et al., 2004; Turriziani et al., 2004; Aggleton et al., 2005; Barbeau et al., 2005; Holdstock et al., 2005), patient PR showed a more consistent impairment of recall than recognition on the experimental test battery. In fact, his recognition was unimpaired on over half of the tests. Coupled with his striking dissociation between significantly impaired recall and apparently completely spared recognition on the standardized Doors and People test, these data show that, although PR's recall is clearly impaired, his recognition memory can be very good.

However, PR's recognition performance was much more variable than that of both the matched controls and patient AC. Furthermore, his recognition performance followed no specific pattern (see Table 2) and so, unlike other cases reported in the literature (Holdstock et al., 2002a; Bastin et al., 2004; Aggleton et al., 2005; Barbeau et al., 2005), cannot easily be explained by a selective sparing of familiarity. For example, he was no more impaired on associative recognition than on item recognition tests and no more impaired on yes/no than forcedchoice item recognition tests, even when targets and corresponding foils were very similar. Both associative recognition and yes/no item recognition, particularly when a fine discrimination is required between targets and very similar foils, have been argued to require recollection and so a patient whose recognition is based primarily on familiarity would be expected to be impaired on these tasks (see Norman and O'Reilly, 2001; O'Reilly and Norman, 2002; Yonelinas, 2002). PR may therefore have some sparing of both recollection and familiarity, despite a consistent and clear deficit in recall. Such a pattern of performance is inconsistent both with the proposals of Squire and Zola-Morgan (1991) and Aggleton and Brown (1999). However, it remains to be determined whether both recollection and familiarity are at normal levels when his recognition performance is very good.

We are not certain why PR performed poorly on some of the recognition tests. Neurological patients, with etiologies of stroke, head injury, and neurodegenerative disorders, have been reported to show greater test-retest variability on the Recognition Memory Test than healthy controls (Bird and Cipolotti, 2007), and so it could be argued that variable performance is a natural consequence of poor memory, and an associated increase in guessing, following neurological damage. However, inconsistent recognition performance has not been reported for previous cases of relatively selective hippocampal damage and so does not appear to be a characteristic of selective hippocampal damage per se. Furthermore, this potential explanation, which implies that PR's good performance is due to guessing, does not account well for PR's pattern of recognition memory performance. PR's performance is often, rather than occasionally, above or within $1.96 \mathrm{SDs}$ of the control mean (9 or the 16 recognition tests) and on a number of these tests there is a very low probability of PR obtaining recognition scores as high as he does by chance (guessing) alone (for example, $8.6 \times 10^{-4}$ for forced-choice pattern recognition, $4.2 \times 10^{-4}$ for forced-choice definition recognition $0 \mathrm{~s}$, $3.3 \times 10^{-11}$ for forced-choice word temporal order recognition, $4.2 \times 10^{-4}$ for forced-choice word-definition recognition at $0 \mathrm{~s}$ and 30 s delays, all calculated using the binomial distribution). Rather, the high variability of his performance appears to be driven by two tests on which he performs particularly poorly (11.41 and 7.85 SDs below the control mean). This pattern is more consistent with occasional disruption of a functioning recognition system than increased guessing associated with generally poor memory.

So, what may have intermittently disrupted PR's recognition memory? We consider it unlikely that his performance varied because of a general lack of motivation during the test sessions. PR appeared to try his best on all of the tests administered and, consistent with this impression, he performed extremely well on all of the nonmnemonic tests that he completed (WASI full-scale IQ 129, Trail Making test 75th to 90th percentile, Stroop test $100 \%$ correct). However, given that his recognition performance showed no specific pattern, and that patients in remission from major depression have been reported to show impairments on tests of attention and executive function (Paelecke-Habermann et al., 2005), it remains possible that his variable scores resulted from lapses in attention and concentration throughout the test sessions. We cannot rule out this explanation because we do not have psychometric measures of sustained attention for PR, however, it should be noted that, contrary to the patients reported by Paelecke-Habermann et al. (2005), PR was unimpaired on both of the tests of executive function that he completed, which included the Stroop test on which Paelecke-Habermann's patients were impaired.

Another possibility is that variable recognition performance resulted from partial dysfunction of the perirhinal system. An- 
oxia rarely produces selective hippocampal damage (Caine and Watson, 2000) and given the proximity of the perirhinal cortex to the hippocampus, it would not be surprising for this region to be damaged or dysfunctional. Atrophy to the perirhinal cortex would be expected to disrupt familiarity memory at least intermittently, depending on how much the working efficiency of the neural circuitry relevant to mediating familiarity for each kind of stimulus has been affected. It is therefore of interest to note that even when PR's recognition performance was unimpaired, it was below the control mean on the majority of tests (7 of the 9 recognition tests on which he was unimpaired). This contrasts with patient AC, whose recognition performance was primarily above the control mean. If PR's familiarity memory fluctuates across time, as a result of an intermittent disruption, then this may also partly explain his variable recognition performance. However, it would not be easy to explain his variable performance on recognition tests that are thought to require recollection, such as the yes/no object recognition tests, which required fine discriminations between targets and foils, and the associative recognition tests.

Furthermore, there is no strong evidence from PR's anatomical data in support of this explanation. Although there was some suggestion that he may have damage to the perirhinal cortex, the methods used in our study did not show this convincingly. Structural MRI showed that his perirhinal cortex is smaller than the control mean bilaterally with the volume difference being greater on the right: his left perirhinal cortex was $12 \%$ smaller than the control mean, and his right perirhinal cortex was $30 \%$ smaller than the control mean. However, the volumes were not significantly smaller than the control mean and no convincing evidence of abnormality was detected in either perirhinal cortex by a neuroradiologist from the King's College Hospital, London, who has extensive experience of inspecting MRI scans for temporal lobe abnormality (Joe Jarosz, personal communication). It is also slightly surprising, if disruption outside the hippocampus is primarily to PR's right perirhinal cortex, that his performance on verbal and nonverbal recognition tests was comparable (although this can be explained if verbal mediation was used to help support nonverbal recognition memory on our tasks). Nevertheless, it should be noted that assessing perirhinal cortex damage and dysfunction is at the limits of current techniques so that subtle changes to the structure or function of this region may not always be detected. In relation to this, it should be noted that high variability of perirhinal cortex volumes in our control subjects made it very hard to establish whether PR's small right perirhinal volume was due to atrophy or natural variation within the normal population. As a result of these limitations, we cannot rule out the possibility that PR has damage or dysfunction to the perirhinal cortex that is contributing to his memory deficit. This case highlights the importance of overcoming these limitations in future studies, which may be achieved by employing improved structural volume measures that make use of both $\mathrm{T} 1$ and $\mathrm{T} 2$ weighted $3 \mathrm{D}$ images, as well as making measures of brain function.

Patient AC showed a very different pattern of performance to patient PR and to the previously reported cases in the litera- ture. Unlike these other patients he showed no memory deficits despite a mean bilateral hippocampal volume reduction of $28 \%$, which was comparable to a number of the previously reported cases. Both recall and recognition were unimpaired. This was the case even for the word-definition learning test which appears to be a very sensitive measure of recall, and on which PR performed 50 SDs below the control mean after the tenth learning trial. AC's performance hovered around the control mean on this test $(0.23$ SDs below the control mean on the tenth learning trial and 0.23 SDs above the control mean on the preceding trial).

Patient AC's performance is therefore very unusual, but this may relate, at least in part, to the way in which patients are selected for inclusion in studies of hippocampal function. Often patients with bilateral hippocampal lesions are identified because they experience memory problems in daily life and are undergoing assessment or rehabilitation as a result. In contrast, patients who do not experience any cognitive deficits are less likely to come to the attention of researchers. As patient AC's data indicate, such a bias in patient selection is likely to result in an incomplete and potentially biased account of the contribution of the hippocampus to memory. Future studies need to select patients on the basis of their brain lesion, that is, MRI confirmed selective hippocampal damage, irrespective of the status of their memory, if a complete understanding about when and why hippocampal lesions result in global or more selective memory deficits is to emerge.

In relation to this aim, AC's preserved memory is extremely interesting particularly when compared with that of the patients investigated by Squire et al. (e.g., Reed and Squire, 1997; Stark et al., 2002; Stark and Squire, 2003) who suffered a severe global anterograde amnesia, that affected recognition as much as recall, after hippocampal volume reductions of between 10 and 45\% (Manns et al., 2003; Stark and Squire, 2003). In general, these patients had smaller reductions in hippocampal volume than the patients who have been reported in the literature to have preserved recognition memory (see Mayes et al., 2002). It has therefore been suggested that difference in lesion size may explain the differences in recognition performance between patients (Mayes et al., 2002; Barbeau et al., 2005). In particular, based on the results of nonhuman primate work using the delayed nonmatching to sample task (Baxter and Murray, 2001a,b; but see also Zola and Squire, 2001), it has been argued that there may be an inverse relationship between size of hippocampal lesion and recognition memory deficit, such that, smaller hippocampal lesions result in greater recognition memory deficits (Mayes et al., 2002; Barbeau et al., 2005). However, AC's hippocampus was reduced in volume by a very similar amount to the patients who suffered global amnesia [JRW 29\%, GW 45\%, RS 40\%, MJ 10\%, LJ 29\%, HC 27\%, PH 30\%, Manns et al. (2003), Stark and Squire (2003)] and yet both his recognition and recall were unimpaired. This indicates that a simple explanation in terms of differences in lesion size cannot completely explain the heterogeneity in the patterns of memory performance that have been reported after hippocampal damage. Other factors must also play a role and may 
fully explain the heterogeneity. It should also be noted that two of the patients with global anterograde amnesia have high IQs of a similar level to AC (MJ WAIS III Full-scale IQ 139 and $10 \%$ hippocampal volume reduction; PH WAIS III Full-scale IQ 115 and 30\% hippocampal volume reduction), which suggests that it is unlikely that AC's high IQ "protected" his memory so that it remained at normal levels.

AC's preserved memory performance shows that a mean bilateral hippocampal volume loss of $28 \%$ is not sufficient to produce a deficit in recall and recognition memory in patients with adult-onset anoxic brain injury. Other factors such as the location and nature of the lesion within the hippocampus, the functionality of the remaining hippocampal tissue, and dysfunction outside the hippocampus (which may or may not be related to extra-hippocampal damage) must be important in determining the nature of the memory deficit suffered by any specific patient (see Mayes et al., 2002). Similar suggestions have been made regarding developmental amnesia. Isaacs et al. (2003) suggest that a 20 to $30 \%$ bilateral hippocampal volume loss is necessary, but may not be sufficient, for the occurrence of this disorder, and that pathology in other brain regions, including that identified in the putamen, posterior thalamus and retrosplenial cortex, as well as the functionality of the remaining hippocampal tissue, may be important.

The imaging data available for our and the other patients with adult-onset hippocampal lesions in the literature do not allow these issues to be fully explored at present. As discussed earlier, in relation to patient PR, improved imaging of regions adjacent to the hippocampus, such as perirhinal cortex, will be important in future studies to provide stronger evidence of the selectivity of lesions to the hippocampus. Patient AC's data indicate that it will also be important to improve the precision with which lesions can be located within the hippocampus itself. This may be helped by the development of high resolution structural MRI (perhaps using a 7T MRI scanner) and the potential use of techniques such as hippocampal flat mapping (Zeineh et al., 2001). Finally, it will be important for future studies to use functional as well as structural MRI to investigate whether any remaining hippocampal tissue is functional, the presence of brain dysfunction outside the hippocampus and reorganization of brain function. For example, patient AC's memory was initially poor following anoxia but has recovered over time to a point where he no longer complains of memory problems and performs normally on formal tests of memory. Functional imaging data would help determine whether his now normal memory is mediated by the same or by partially different brain systems to that supporting memory in healthy individuals, that is, whether his recovery has occurred because of reorganization of function. The utility of functional imaging in such cases has been demonstrated by Maguire et al. who found that, like controls, the young developmental case, Jon, showed activation of the hippocampus during retrieval of factual information whereas patient VC who had hippocampal damage of adult onset and who had a more global amnesia, did not show hippocampal activation during this task (Maguire et al., 2001, 2005). Furthermore, Jon, unlike both controls and VC, showed bilateral activation of a network of brain regions during factual retrieval. These functional imaging studies therefore revealed differences in the brain regions engaged by the two patients during a memory task which they could both perform successfully, suggesting that, despite having similar structural brain damage, their residual memory was being supported by at least partially different brain systems.

Studying the effect of hippocampal lesions in humans relies on clinical cases who vary in both etiology and the extent of their lesions, and so it is perhaps not that surprising that the patterns of memory deficits that have been reported have been heterogeneous and less consistent than those from animal studies where there is greater control over the extent and location of the lesion. Studying such cases is, however, critical to determine the extent to which findings from the animal literature generalize to humans, and currently provides the best platform for exploring theoretical issues about lesion effects on recollection and familiarity. In both respects, these studies have been informative and have moved forward theoretical thinking about the role of the human hippocampus in memory. The addition of new cases to the literature remains important to further enrich the dataset on which theoretical work can be based. However, given that, as illustrated here, patients who have selective hippocampal damage, according to structural MRI, can show quite strikingly different patterns of memory performance, future studies will need to adopt more sophisticated imaging techniques if substantial further progress is to be made in understanding the role of the hippocampus in human memory.

\section{REFERENCES}

Abrahams S, Morris RG, Polkey CE, Jarosz JM, Cox T, Graves M, Pickering A. 1999. Hippocampal involvement in spatial and working memory: A structural MRI analysis of patients with unilateral mesial temporal lobe sclerosis. Brain Cogn 41:39-65.

Aggleton JP, Brown M. 1999. Episodic memory, amnesia and the hippocampal-anterior thalamic axis. Behav Brain Sci 22:425490.

Aggleton JP, Vann SD, Denby C, Dix S, Mayes AR, Roberts N, Yonelinas AP. 2005. Sparing of the familiarity component of recognition memory in a patient with hippocampal pathology. Neuropsychologia 43:1810-1823.

Baddeley A, Emslie H, Nimmo-Smith I. 1994. Doors and People. England: Thames Valley Test Company.

Barbeau EJ, Felician O, Joubert S, Sontheimer A, Ceccaldi M, Poncet M. 2005. Preserved visual recognition memory in an amnesic patient with hippocampal lesions. Hippocampus 15:587-596.

Bastin C, Van der Linden M, Charnallet A, Denby C, Montaldi D, Roberts N, Mayes AR. 2004. Dissociation between recall and recognition memory performance in an amnesic patient with hippocampal damage following carbon monoxide poisoning. Neurocase 10:330-344.

Baxter MG, Murray EA. 2001a. Opposite relationship of hippocampal and rhinal cortex damage to delayed nonmatching-to-sample deficits in monkeys. Hippocampus 11:61-71. 
Baxter MG, Murray EA. 2001b. Effects of hippocampal lesions on delayed nonmatching-to-sample in monkeys: A reply to Zola and Squire (2001). Hippocampus 11:201-203.

Bird CM, Cipolotti L. 2007. The utility of the recognition memory test and the graded naming test for monitoring neurological patients. Br J Clin Psychol 46:223-234.

Bird CM, Shallice T, Cipolotti L. 2007a. Fractionation of memory in medial temporal lobe amnesia. Neuropsychologia 45:1160-1171.

Bird CM, Vargha-Khadem F, Burgess N. 2007b. Impaired memory for scenes but not faces in developmental hippocampal amnesia: A case study. Neuropsychologia (in press).

Caine D, Watson JDG. 2000. Neuropsychological and neuropathological sequelae of cerebral anoxia: A critical review. J Int Neuropsychol Soc 6:86-99.

Cipolotti L, Shallice T, Chan D, Fox N, Scahill R, Harrison G, Stevens J, Rudge P. 2001. Long-term retrograde amnesia... the crucial role of the hippocampus. Neuropsychologia 39:151-172.

Cipolotti L, Bird C, Good T, Macmanus D, Rudge P, Shallice T. 2006. Recollection and familiarity in dense hippocampal amnesia: A case study. Neuropsychologia 44:489-506.

Crawford JR, Garthwaite PH. 2002. Investigation of the single case in neuropsychology: Confidence limits on the abnormality of test scores and test score differences. Neuropsychologia 40:11961208.

Crawford JR, Howell DC. 1998. Comparing an individual's test score against norms derived from small samples. Clinl Neuropsychol $12: 482-486$.

Gold JJ, Hopkins RO, Squire LR. 2006. Single-item memory, associative memory and the human hippocampus. Learn Mem 13:644649.

Graham KS, Scahill VL, Hornberger M, Barense MD, Lee ACH, Bussey TJ, Saksida LM. 2006. Abnormal categorization and perceptual learning in patients with hippocampal damage. J Neurosci 26:7547-7554.

Henke K, Kroll NEA, Behniea H, Amaral DG, Miller MB, Rafal R, Gazzaniga MS. 1999. Memory lost and regained following bilateral hippocampal damage. J Cogn Neurosci 11:682-697.

Holdstock JS, Mayes AR, Roberts N, Cezayirli E, Isaac CL, O’Reilly R, Norman K. 2002a. Under what conditions is recognition spared relative to recall after selective hippocampal damage in humans? Hippocampus 12:341-351.

Holdstock JS, Mayes AR, Isaac CL, Gong Q, Roberts N. 2002b. Differential involvement of the hippocampus and temporal lobe cortices in rapid and slow learning of new semantic information. Neuropsychologia 40:748-768.

Holdstock JS, Mayes AR, Gong QY, Roberts N, Kapur N. 2005. Item recognition is less impaired than recall and associative recognition in a patient with selective hippocampal damage. Hippocampus 15:203-215.

Insausti R, Kirsi J, Soinimen H, Insausti AM, Partanen K, Vainio P, Laakso MP, Pitkänen A. 1998. MR volumetric analysis of the human entorhinal, perirhinal and temporopolar cortices. Am J of Neuroradiol 19:659-671.

Isaac CL, Mayes AR. 1999. Rate of forgetting in amnesia. I. Recall and recognition of prose. J Exp Psychol [Learn Mem Cogn] 25:942-962.

Isaacs EB, Vargha-Khadem F, Watkins KE, Lucas A, Mishkin M, Gadian DG. 2003. Developmental amnesia and its relationship to degree of hippocampal atrophy. Proc Natl Acad Sci USA 100:13060-13063.

Jack CR, Twomey CK, Zinzmmeister AR, Sharbrough FW, Petersen RC, Cascino GD. 1989. Anterior temporal lobes and hippocampal formations: Normative volumetric measurements from MR images in young adults. Radiology 172:549-554.

Jacoby LL. 1991. A process dissociation framework: Separating automatic from intentional uses of memory. J Mem Lang 30:513541.
Kartsounis LD, Rudge P, Stevens JM. 1995. Bilateral lesions of CA1 and CA2 fields of the hippocampus are sufficient to cause a severe amnesic syndrome in humans. J Neurol Neurosurg Psychiatry 59:95-98.

Lee ACH, Buckley MJ, Pegman SJ, Spiers H, Scahill V, Gaffan D, Bussey TJ, Davies RR, Kapur N, Hodges JR, Graham KS. 2005. Specialization in the medial temporal lobe for processing of objects and scenes. Hippocampus 15:782-797.

Maguire EA, Vargha-Khadem F, Mishkin M. 2001. The effects of bilateral hippocampal damage on fMRI regional activations and interactions during memory retrieval. Brain 124:11561170.

Maguire EA, Frith CD, Rudge P, Cipolotti L. 2005. The effect of adult-acquired hippocampal damage on memory retrieval: An fMRI study. NeuroImage 27:146-152.

Mandler G. 1980. Recognizing: The judgement of previous occurrence. Psychol Rev 87:252-271.

Manns JR, Hopkins RO, Reed JM, Kitchener EG, Squire LR. 2003. Recognition memory and the human hippocampus. Neuron 37:171-180.

Mayes AR, Isaac CL, Downes JJ, Holdstock JS, Hunkin NM, Montaldi D, MacDonald C, Cezayirli E, Roberts JN. 2001. Memory for single items, word pairs, and temporal order in a patient with selective hippocampal lesions. Cogn Neuropsychol 18:97-123.

Mayes AR, Holdstock JS, Isaac CL, Hunkin NM, Roberts N. 2002. Relative sparing of item recognition memory in a patient with adult-onset damage limited to the hippocampus. Hippocampus 12:325-340.

Mayes AR, Holdstock JS, Isaac CL, Montaldi D, Grigor J, Gummer A, Cariga P, Downes JJ, Tsivilis D, Gaffan D, Norman KA. 2004. Associative recognition in a patient with selective hippocampal lesions and relatively normal item recognition. Hippocampus 14:763-784.

Norman KA, O'Reilly RC. 2001. Modeling hippocampal and neocortical contributions to recognition memory: A complementary learning systems approach (ICS Technical report 01-02). Boulder, CO: University of Colorado, Institute of Cognitive Science.

O'Reilly RC, Norman KA. 2002. Hippocampal and neocortical contributions to memory: Advances in the complementary learning systems framework. Trends Cogn Sci 6:505-510.

Paelecke-Habermann Y, Pohl J, Leplow B. 2005. Attention and executive functions in remitted major depression patients. J Affect Disord 89:125-135.

Parslow DM, Morris RG, Fleminger S, Rahman Q, Abrahams S, Recce M. 2005. Allocentric spatial memory in humans with hippocampal lesions. Acta Psychol 118:123-147.

Pollack L, Norman DA. 1964. Non-parametric analysis of recognition experiments. Psychon Sci 1:125-126.

Reed JM, Squire LR. 1997. Impaired recognition memory in patients with lesions limited to the hippocampal formation. Behav Neurosci 111:667-675.

Scoville WB, Milner B. 1957. Loss of recent memory after bilateral hippocampal lesions. J Neurol Neurosurg Psychiatry 20:11-21.

Sheline YI, Mittler BL, Mintun MA. 2002. The hippocampus and depression. Eur Psychiatry 17:300-305.

Squire LR, Zola-Morgan S. 1991. The medial temporal-lobe memory system. Science 253:1380-1386.

Stark CEL, Bayley PJ, Squire LR. 2002. Recognition memory for single items and for associations is similarly impaired following damage to the hippocampal region. Learn Mem 9:238242.

Stark CEL, Squire LR. 2003. Hippocampal damage equally impairs memory for single items and memory for conjunctions. Hippocampus 13:281-292. 
Turriziani P, Fadda L, Caltagirone C, Carlesimo GA. 2004. Recognition memory for single items and for associations in amnesic patients. Neuropsychologia 42:426-433.

Vargha-Khadem F, Gadian DG, Watkins KE, Connelly A, van Paesschen W, Mishkin M. 1997. Differential effects of early hippocampal pathology on episodic and semantic memory. Science 277:376-380.

Wais PE, Wixted JT, Hopkins RO, Squire LR. 2006. The hippocampus supports both the recollection and the familiarity components of recognition memory. Neuron 49:459-466.

Yonelinas AP. 2002. The nature of recollection and familiarity: A review of 30 years of research. J Mem Lang 46:441-517.
Yonelinas AP, Kroll NEA, Quamme JR, Lazzara MM, Sauvé MJ, Widaman KF, Knight RT. 2002. Effects of extensive temporal lobe damage or mild hypoxia on recollection and familiarity. Nat Neurosci 5:1236-1241.

Zeineh MM, Engel SA, Thompson PM, Bookheimer SY. 2001. Unfolding the human hippocampus with high resolution structural and functional MRI. Anat Rec (New Anat) 265:111-120.

Zola SM, Squire LR. 2001. Relationship between magnitude of damage to the hippocampus and impaired recognition memory in monkeys. Hippocampus 11:92-98. 